\title{
A Decomposition of 2-Weak Vertex-Packing Polytopes*
}

\author{
E. Steingrímsson
}

Matematiska Institutionen CTH \& GU,

41296 Göteborg, Sweden

einar@math.chalmers.se

\begin{abstract}
The 2-weak vertex-packing polytope of a loopless graph $G$ with $d$ vertices is the subset of the unit $d$-cube satisfying $x_{i}+x_{j} \leq 1$ for every edge $(i, j)$ of $G$. The dilation by 2 of this polytope is a polytope $\mathscr{P}$ with integral vertices. We triangulate $\mathscr{P}$ with lattice simplices of minimal volume and label the maximal simplices with elements of the hyperoctahedral group $B_{d}$. This labeling gives rise to a shelling of the triangulation $\hat{\mathscr{P}}$ of $\mathscr{P}$, where the $h$-vector of $\hat{\mathscr{P}}$ (and the Ehrhart $h^{*}$-vector of $\mathscr{P}$ ) can be computed as a descent statistic on a subset of $B_{d}$ defined in terms of $G$. A recursive way of computing the $h$-vector of $\hat{\mathscr{P}}$ is also given, and a recursive formula for the volume of $\mathscr{P}$.
\end{abstract}

\section{Introduction}

Let $G$ be a graph (with no loops), let $d$ be the number of vertices in $G$, and label the vertices of $G$ by the integers $1,2, \ldots, d$. The vertex-packing polytope of $G$ is the $d$-polytope $\mathscr{V}(G)$ in $\mathbf{R}^{d}$ defined by the following inequalities:

$$
\begin{gathered}
0 \leq x_{i} \leq 1, \quad 1 \leq i \leq d, \\
x_{i_{1}}+x_{i_{2}}+\cdots+x_{i_{k}} \leq 1 \quad \text { if }\left\{i_{1}, i_{2}, \ldots, i_{k}\right\} \text { is a clique of } G .
\end{gathered}
$$

These polytopes have been studied by various authors, mostly from the linear programming point of view (for some background, see [4] or [8]). A simpler

\footnotetext{
* This work was partially supported by grants from the Icelandic Council of Science and the Royal
} Swedish Academy of Sciences, respectively. 
polytope, related to $\mathscr{V}(G)$, is the 2-weak vertex-packing polytope of $G$, which we denote by $\mathscr{W}(G)$ and which is defined by these inequalities:

$$
\begin{gathered}
x_{i} \leq 1, \quad 1 \leq i \leq d, \\
0 \leq x_{i}, \quad 1 \leq i \leq d, \\
x_{i}+x_{j} \leq 1 \quad \text { if }(i, j) \text { is an edge of } G .
\end{gathered}
$$

If $G$ is a bipartite graph, then $\mathscr{W}(G)$ and $\mathscr{V}(G)$ coincide.

It is easily seen that the inequalities given by (4) and (5) and those of the inequalities (3) for which $i$ is an isolated vertex of $G$ are linearly independent, and thus these inequalities define the facets of $\mathscr{W}(G)$. We will show that the vertices of $\mathscr{W}(G)$ have coordinates in $\left\{0, \frac{1}{2}, 1\right\}$. Hence, if we dilate $\mathscr{W}(G)$ by 2 , i.e., take the image of $\mathscr{W}(G)$ under the map $f: \mathbf{R}^{d} \rightarrow \mathbf{R}^{d}$ defined by $f(x)=2 x$, then we get a polytope $\mathscr{P}(G)$ whose vertices are integral, more precisely $\{0,1,2\}$-valued. We call the polytope $\mathscr{P}(G)$ the extended 2-weak vertex-packing polytope of $G$. It is clearly defined by

$$
\begin{gathered}
x_{i} \leq 2, \quad 1 \leq i \leq d, \\
0 \leq x_{i}, \quad 1 \leq i \leq d, \\
x_{i}+x_{j} \leq 2 \quad \text { if }(i, j) \text { is an edge of } G .
\end{gathered}
$$

This paper deals with the structure of $\mathscr{P}(G)$. We triangulate $\mathscr{P}(G)$ in a certain systematic way and label the maximal simplices in the triangulation, which we denote by $\widehat{\mathscr{P}}$, with elements of the hyperoctahedral group $B_{d}$. This labeling allows us to shell $\widehat{\mathscr{P}}$ in such a way that we can compute the $h$-vector of $\mathscr{P}(G)$ as a descent statistic on a subset of $B_{d}$ determined by $G$. Moreover, the triangulation is such that its $h$-vector equals the Ehrhart $h^{*}$-vector of $\mathscr{P}(G)$. We give a decomposition of $\hat{\mathscr{P}}$ into maximal simplices, whose intersections with other maximal simplices we can describe explicitly. We also give a recursive formula for computing the $h$-vector of $\mathscr{P}(G)$, be descending to those facets of $\mathscr{P}(G)$ which lie on the boundary of $2 C^{d}$ where $C^{d}$ is the standard unit $d$-cube (these facets are extended 2-weak vertex-packing polytopes in their own right). A simplified version of this recursion gives a simple recursive formula for computing the volume of $\mathscr{P}(G)$.

\section{Preliminaries}

\subsection{Ehrhart Polynomials}

Let $\mathscr{P}$ be a $d$-dimensional polytope (or simplical complex (see Section 12.2)) in $\mathbf{R}^{\boldsymbol{n}}$ with integral (or lattice) vertices, i.e., $v_{i} \in \mathbf{Z}^{n}$ for all vertices $v_{i}$ of $\mathscr{P}$. For $k \in \mathbf{N}$ let 
$k \mathscr{P}=\{k x \mid x \in \mathscr{P}\}$, i.e., $k \mathscr{P}$ is the (lattice) polytope obtained by dilating $\mathscr{P}$ by a factor of $k$.

For $k \in \mathbf{N}$ define the function

$$
i(\mathscr{P P}, k)=\#\left\{x \in \mathbf{R}^{n} \mid x \in k \mathscr{P} \cap \mathbf{Z}^{n}\right\}
$$

Thus, $i(\mathscr{P}, k)$ is the number of lattice points contained in $k \mathscr{P}$. By Corollary 4.6 .28 in [11], $i(\mathscr{P}, k)$ is a polynomial in $k$ of degree $d$, called the Ehrhart polynomial of $\mathscr{P}$. Now define the generating function

$$
E(\mathscr{P}, t)=\sum_{k \geq 0} i(\mathscr{P}, k) t^{k}
$$

By Theorem 2.1 in [10], we have

$$
E(\mathscr{P}, t)=\frac{h^{*}(\mathscr{P}, t)}{(1-t)^{d+1}}
$$

where $h^{*}(\mathscr{P P}, t)$ is a polynomial of degree at most $d$ with nonnegative integer coefficients, called the Ehrhart $h^{*}$-polynomial of $\mathscr{P}$. Its coefficients are the coordinates of the Ehrhart $h^{*}$-vector $h^{*}(\mathscr{P})$ of $\mathscr{P}$.

\subsection{Simplical Complexes}

An abstract simplical complex is a nonempty collection $K$ of sets such that if $F \in K$ and $G \subset F$, then $G \in K$. An element of $K$ is called a face of $K$. We are mostly concerned with the geometric realization of simplical complexes (for definitions and basic properties, see [7]) and, by abuse of notation, we do not distinguish between a simplical complex and its geometric realization.

A simplicial complex $K$ is pure if all its maximal faces have the same dimension $d=\operatorname{dim}(K)$. If $K$ is a pure simplicial complex of dimension $d$, then a facet of $K$ is a $d$-face, i.e., a $d$-dimensional face, of $K$. In other words, a facet is a maximal simplex of $K$. When a complex $K$ triangulates a polytope $\mathscr{P}$, the facets of $K$ are $d$-dimensional, but the facets of $\mathscr{P}$ have dimension $d-1$. This can cause confusion, which we try to avoid in subsequent sections by referring to the facets of $K$ as maximal simplices.

The $h$-vector $h(K)=\left(h_{0}, h_{1}, \ldots, h_{d}\right)$ of a simplicial complex $K$ of dimension $d-1$ is defined as follows: Let $f_{1}=f_{i}(K)$ be the number of $i$-dimensional faces in $K$, where we set $f_{-1}=1$ (corresponding to the empty set), and define $h(K)=$ $\left(h_{0}, h_{1}, \ldots, h_{\mathrm{d}}\right)$ by setting

$$
\sum_{i=0}^{d} f_{i-1}(x-1)^{d-i}=\sum_{i=0}^{d} h_{i} x^{d-i}
$$


We define the $h$-polynomial $h(K, t)$ of $K$ by $h(K, t)=h_{0}+h_{1} t+\cdots+h_{d} t^{d}$. For further information about $h$-vectors, see [13].

Let $K$ be a pure simplicial lattice complex of dimension $d$. If all facets of $K$ have volume $1 / d$ ! (see Section 2.4 ), then we say that $K$ is primitively triangulated. The following theorem is essentially a consequence of Corollary 2.5 in [10], whose conclusion is expressed in greater generality in Theorem 2 in [1].

Theorem 1. Suppose $K$ is a primitively triangulated simplicial lattice complex. Then $h^{*}(K, t)=h(K, t)$, where $h^{*}(K, t)$ is the Ehrhart $h^{*}$-polynomial of $K$.

For certain pure simplicial complexes $K$ the coefficients of $h(K, t)$ can be interpreted in a way that partitions the facets of $K$ according to how they intersect other facets. We briefly review this now. For further information, see [2] and [3].

Definition 2. Let $K$ be a finite pure simplicial complex of dimension $d$. If $F$ is a face of $K$, let $\bar{F}$ be the complex consisting of $F$ and all its faces. An ordering $F_{1}, F_{2}, \ldots, F_{n}$ of the facets of $K$ is called a shelling if, for all $k$ with $1<k \leq n$, $\bar{F}_{k} \cap \bigcup_{i<k} \bar{F}_{i}$ is a nonempty union of $(d-1)$-faces of $\bar{F}_{k}$. A complex $K$ is said to be shellable if a shelling of $K$ exists.

That is, a complex is shellable if it can be built up by adding one facet at a time in such a way that, for $k>1$, the intersection of each $\bar{F}_{k}$ with the complex generated by the previous $\bar{F}_{i}$ 's is a nonempty union of $(d-1)$-faces of $\bar{F}_{k}$.

As it turns out, the $h$-vector of a shellable complex can be computed from the shelling. The following theorem is essentially due to McMullen [6].

Theorem 3. Let $F_{1}, F_{2}, \ldots, F_{n}$ be a shelling of a d-dimensional complex $K$ and let $c(k)$ be the number of $(d-1)$-faces of $\bar{F}_{k}$ contained in $\bigcup_{i<k} \bar{F}_{i}$. Then we have the following formula for the h-polynomial of $K$ :

$$
h(K, t)=\sum_{k=1}^{n} t^{c(k)} .
$$

Thus, given a shelling $F_{1}, F_{2}, \ldots, F_{n}$ of a simplicial complex $K$, we can compute the $h$-polynomial $h(K, t)$ of $K$ via Theorem 3 . That is, the $k$ th coefficient of $h(K, t)$ equals the number of $F_{i}$ with $c(i)=k$. We then say that a facet $F_{i}$ of $K$ contributes to the $k$ th coefficient of $h(K, t)$ if $c(i)=k$.

If $K$ is a simplicial complex and $p$ is a vertex not in $K$, then the cone with apex $p$ over $K$ (or with base $K$ ), denoted $p * K$, is the simplicial complex whose $i$-faces are the $i$-faces of $K$ and $\{p \cup f \mid f$ an $(i-1)$-face of $K\}$. Geometrically, a cone can be defined as follows. If $K$ is a $(d-1)$-dimensional simplicial (or polytopal) complex in $\mathbf{R}^{n}$ and $p$ is a point in $\mathbf{R}^{n}$ such that each ray emanating from $p$ intersects $K$ in at most one point, then the cone $p * K$ consists of $K$ and $p$ and the new $i$-faces, for $1 \leq i \leq d$, obtained by taking, for each $(i-1)$-face $f$ in $K$, the union of all line segments connecting $p$ to points in $f$.

The following theorem is straightforward to prove from (9). 
Theorem 4. Suppose the simplicial complex $K$ is a cone with apex $p$ over $B$, i.e., $K=p * B$. Then $h(K, t)=h(B, t)$.

\subsection{The Hyperoctahedral Group}

The hyperoctahedral group $B_{d}$ is the group of symmetries of the $d$-dimensional cross polytope (or hyperoctahedron) defined as the convex hull of the points $\pm e_{i}$ where $e_{i}$ is a standard basis vector in $\mathbf{R}^{d}$. We represent the elements of the hyperoctahedral group $B_{d}$ by signed permutation words, i.e., ordinary permutation words in which each letter has a sign. To simplify the notation, we write $\overline{1}, \overline{2}, \overline{3}$, etc., for $-1,-2,-3$, and simply $1,2,3$, etc., for $+1,+2,+3$. For example, $B_{2}=\{12,21, \overline{1} 2,2 \overline{1}, 1 \overline{2}, \overline{2} 1, \overline{1} \overline{2}, \overline{2} \overline{1}\}$.

As we are not concerned with ordinary permutations, we simply call the elements of $B_{d}$ permutations. When we refer to the letters in a permutation, we regard them as integers (with the sign incorporated) and order them as such, i.e., $\cdots \overline{3}<\overline{2}<\overline{1}<0<1<2<3 \cdots$. Let $[d]:=\{1,2, \ldots, d\}$.

Definition 5. A descent in $\pi \in B_{d}$ is an $i \in[d]$ such that one of the following holds:

1. $i<d$ and $a_{i}>a_{i+1}$.

2. $i=d$ and $a_{d}>0$.

The number of descents in $\pi \in B_{d}$ is $\operatorname{des}(\pi)$. For any subset $S$ of $B_{d}$, the descent polynomial of $S$ is $D(S, t):=\sum_{\pi \in S} t^{\operatorname{des}(\pi)}$.

For example, the descents of $2 \overline{3} \overline{4} 1$ are 1,2 , and 4 , so $\operatorname{des}(2 \overline{3} \overline{4} 1)=3$. If $S=$ $\{\overline{3} \overline{2} 1,1 \overline{2} \overline{3}, 213\}$, then $D(S, t)=1+2 t^{2}$.

For more information on descent (and other) statistics of $B_{d}$, see [9] or [14].

\subsection{Volumes}

When we talk about volume in $\mathbf{R}^{d}$ we mean the usual $d$-dimensional volume, which we denote $\operatorname{vol}_{d}(\cdot)$. If $S$ is a subset of a $d$-dimensional coordinate subspace of $\mathbf{R}^{\boldsymbol{n}}$, then by $\operatorname{vol}_{d}(S)$ we mean the volume of $S$ in that subspace. If $S$ is a union of such subsets $S_{i}$, then by $\operatorname{vol}_{d}(S)$ we mean the sum of the volumes of the $S_{i}$. In particular, a polytope $\mathscr{P}$ of dimension less than $d$ has $\operatorname{vol}_{d}(\mathscr{P})=0$. For convenience, we make the following definition.

Definition 6. If $\mathscr{P}$ is a $d$-dimensional polytope or simplicial complex in $\mathbf{R}^{n}$ such that $\operatorname{vol}_{d}(\mathscr{P})$ is defined, then the normalized volume of $\mathscr{P}$ is $\mathrm{Nvol}(\mathscr{P}):=d ! \cdot \operatorname{vol}_{d}(\mathscr{P})$.

Hence, for any polytope (or simplicial complex) $\mathscr{P}$ of positive dimension, $\operatorname{Nvol}(\mathscr{P})$ is positive. The rationale behind this definition is that the least volume a 
lattice $d$-simplex can have is $1 / d$ !. In particular, the normalized volume of a primitively triangulated complex equals its number of maximal simplices.

\section{Main Theorems}

The following proposition is intuitively obvious, but we give a proof anyway.

Proposition 7. Let $\mathbf{p}$ be a point in the polytope $\mathscr{P}$ and let $\mathscr{P}_{\mathrm{p}}$ be the union of those facets of $\mathscr{P}$ which do not contain $\mathbf{p}$. The $\mathscr{P}$ is a cone with apex $\mathbf{p}$ over $\mathscr{P}_{\mathbf{p}}$.

Proof. If $\mathbf{p}$ is contained in the interior of $\mathscr{P}$ this is trivial, and the same is true if the dimension of $\mathscr{P}$ is 1 . Also, by convexity, any ray from $\mathbf{p}$ can intersect $\mathscr{P}_{\mathbf{p}}$ in at most one point.

Assume, then, that $\mathbf{p}$ lies in some facet of $\mathscr{P}$ and $\operatorname{dim}(\mathscr{P}) \geq 2$. Let $\mathbf{x}$ be a point in $\mathscr{P}$. It suffices to show that $\mathbf{x}$ lies on a line segment between $\mathbf{p}$ and a point $q \in G$ for some facet $G \subset \mathscr{P}_{\mathbf{p}}$. Let $R$ be the extension beyond $\mathbf{x}$ of the line segment px and let $\mathbf{q}$ be the point on $R$ such that every point on $R$ not on pq lies outside $\mathscr{P}$.

It is clear that if $\mathbf{x}$ does not belong to any of the facets of $\mathscr{P}$ containing $\mathbf{p}$, then neither does $q$, by convexity, and we would be done. Suppose, therefore, that $\mathbf{x}$ and $\mathbf{p}$ belong to the same facet $F$ of $\mathscr{P}$. We now proceed by induction, so we may further assume that $\mathbf{q}$ belongs to a $(d-2)$-face $f$ of $F$ such that $\mathbf{p} \notin f$. Let $G$ be the unique facet of $\mathscr{P}$ different from $F$ and containing $f$. We claim that $G$ does not contain p, which will complete the proof. If $G$ did contain p, then $G \cap F$ would contain the $(d-1)$-dimensional set $\mathbf{p} * f$, which is absurd.

Throughout this paper, given a graph $G, \mathscr{P}(G)$ is the extended 2-weak vertexpacking polytope of $G$. It is clear from the definition of $\mathscr{P}(G)$ that it is a subset of $2 C^{d}$, the dilation of the unit $d$-cube by 2 .

Theorem 8. Let $G$ be a graph and let $\mathscr{P}^{\prime}(G)=\mathscr{P}(G) \cap \partial\left(2 C^{d}\right)$, i.e., $\mathscr{P}^{\prime}(G)$ is the union of those facets of $\mathscr{P}(G)$ which lie on the boundary of $2 C^{d}$. Let $\mathbf{p}=(1,1, \ldots, 1)$. Then $\mathscr{P}(G)=\mathbf{p} * \mathscr{P}^{\prime}(G)$.

Proof. The facets of $\mathscr{P}(G)$ are defined by inequalities (7) and (8) and those of inequalities (6) for which $i$ is an isolated vertex in $G$ (or, more precisely, by the corresponding boundary equalities). Hence, those facets of $\mathscr{P}(G)$ which do not lie on $\partial\left(2 C^{d}\right)$ are precisely those which are defined by $x_{i}+x_{j}=2$ for some $(i, j)$. Every such facet contains the point $p$ and no other facets of $\mathscr{P}(G)$ do. Hence, by Proposition $7, \mathscr{P}(G)=\mathbf{p} * \mathscr{P}^{\prime}(G)$.

To triangulate $\mathscr{P}(G)$ we first triangulate $2 C^{d}$ in the following way. $2 C^{d}$ is embedded in $\mathbf{R}^{d}$ so that its vertices are all points whose coordinates are either 0 or 2. In particular, its center (of symmetry) is the point $p=(1,1, \ldots, 1)$. We subdivide $2 C^{d}$ into the $2^{d}$ unit cubes all of whose vertices are lattice points. Each 
of these small cubes contains $\mathbf{p}$ and a unique vertex which is a vertex of $2 C^{d}$. We label each small cube by that vertex of $2 C^{d}$ which it contains. As an example, the standard unit $d$-cube is labeled by $\mathbf{0}=(0,0, \ldots, 0)$ and denoted $c_{0}$.

Next, we triangulate each of these small cubes. Let $c_{z}$ be the small cube labeled by $z$. Then every maximal simplex in the triangulation of $c_{z}$ contains $p$ and $z$ and is defined as the convex hull of a path along edges of $c_{\mathbf{z}}$ from $\mathbf{p}$ to $\mathbf{z}$, as follows.

Let $\mathbf{p}_{0}=\mathbf{p}, \mathbf{p}_{1}, \mathbf{p}_{2}, \ldots, \mathbf{p}_{d}=\mathbf{z}$ be a sequence of vertices of $c_{\mathbf{z}}$ such that $\mathbf{p}_{k}=$ $\mathbf{p}_{k-1} \pm e_{j}$ where $e_{j}$ is the vector $(0, \ldots, 0,1,0, \ldots, 0)$ with a 1 in the $j$ th place and 0 's elsewhere. It follows that in the sequence of $\mathbf{p}_{k}$ 's the $i$ th coordinate must change precisely once, from 1 to $z_{i}$, because we start out from $p=(1,1, \ldots, 1)$ and $\mathbf{z}=\left(z_{1}, z_{2}, \ldots, z_{d}\right)$ is a vertex of $2 C^{d}$, so $z_{i} \in\{0,2\}$ for each $i$.

The points $\mathbf{p}_{i}$ are easily seen to be geometrically independent and thus they are the vertices of a $d$-dimensional simplex, namely, their convex hull. It is also clear that the intersection of any two maximal simplices is the convex hull of their common vertices, so this is indeed a simplicial complex. (In the case of the unit $d$-cube, this triangulation is well known. See, for example, [12].)

Such a sequence of vertices defining a maximal simplex can be coded by a permutation in the hyperoctahedral group $B_{d}$. Namely, we define $\pi=a_{1} a_{2} \cdots a_{d}$ by setting $a_{i}=k$ if $\mathbf{p}_{i}-\mathbf{p}_{i-1}=e_{k}$ and $a_{i}=-k$ if $\mathbf{p}_{i}-\mathbf{p}_{i-1}=-e_{k}$. For example, the sequence $(1,1,1),(0,1,1),(0,1,2),(0,0,2)$ of points in $c_{(0,0,2)}$ corresponds to the permutation 132 . Conversely, every $\pi \in B_{d}$ determines a unique path from $\mathbf{p}$ to a vertex $\mathbf{z}$ of $2 C^{d}$ and hence a unique $d$-simplex, which we denote by $\sigma_{n}$, contained in $c_{z}$. The number of distinct paths from $\mathbf{p}$ to $\mathbf{z}$ is $d !$, and the following lemma is now immediate.

Lemma 9. Let $\left\{\pi_{i} \mid 1 \leq i \leq d\right.$ ! $\}$ be the permutations labeling the maximal simplices in a cube $c_{z}$. Then each integer $k$ in [d] appears with the same sign in every $\pi_{i}$. More precisely, the sign of $k \in[d]$ in such a permutation is + or - according to whether the $k$ th coordinate of $\mathrm{z}$ is 2 or 0 . Conversely, if each $k \in[d]$ appears with the same sign in two permutations $\pi$ and $\tau$, then $\sigma_{\pi}$ and $\sigma_{\tau}$ belong to the same cube $c_{z}$.

For example, the paths in the cube $c_{(0,2)}$ are $(1,1) \rightarrow(1,2) \rightarrow(0,2)$ and $(1,1) \rightarrow$ $(0,1) \rightarrow(0,2)$, corresponding to the permutations $2 \overline{1}$ and $\overline{1} 2$, respectively.

Proposition 10. The collection $\left\{\sigma_{\pi} \mid \pi \in B_{d}\right\}$ covers $2 C^{d}$. Any two of these simplices are isometric, in particular each has volume $1 / d$ ! and hence $\operatorname{Nvol}\left(\sigma_{\pi}\right)=1$ for each $\pi$.

Proof. Given a cube $c_{z}$, there is an orientation-preserving isometry which fixes $(1,1, \ldots, 1)$ takes $z$ to 0 , and each vertex of $c_{x}$ to a vertex of $c_{0}$ (there are many such isometries, of course). Such an isometry sends $\sigma_{\pi} \subset c_{z}$ to some $\sigma_{z} \subset c_{0}$. Given $\sigma_{\alpha} \subset c_{0}$, a suitable permutation of the coordinate axes in $\mathbf{R}^{d}$ sends $\sigma_{\tau}$ to $\sigma_{\alpha}$, so $\sigma_{\tau}$ and $\sigma_{\alpha}$ are isometric. If $\sigma_{\tau} \subset c_{0}$, then all the letters of $\tau$ are negative, say $\tau=-a_{1}-a_{2} \cdots-a_{d}$. It follows from the definition of $\sigma_{\tau}$ that

$$
\sigma_{\tau}=\left\{\mathbf{x}=\left(x_{1}, x_{2}, \ldots x_{d}\right) \in \mathbf{R}^{d} \mid 0 \leq x_{a_{1}} \leq x_{a_{2}} \leq \cdots \leq x_{a_{d}} \leq 1\right\}
$$


Clearly, every point in $c_{0}$ is contained in a subset defined by some $\tau$. There are $d$ ! maximal simplices $\sigma_{\tau} \subset c_{0}$ and the intersection of any two such distinct simplices has dimension at most $d-1$, so $\operatorname{vol}_{d}\left(\sigma_{\tau}\right)=1 / d$ !.

Thus the collection $\left\{\sigma_{\pi} \mid \pi \in B_{d}\right\}$ triangulates $2 C^{d}$. We denote this triangulation by $2 C^{d}$. We can now give a succinct characterization of the permutations corresponding to the maximal simplices of $2 C^{d}$ contained in $\mathscr{P}$. First a definition.

Definition 11. Let $G$ be a graph. The set of permissible permutations with respect to $G$ is $\Pi(G)=\left\{\pi \in B_{d} \mid \sigma_{\pi} \subset \mathscr{P}(G)\right\}$. A permutation $\pi$ is permissible with respect to $G$ if $\pi \in \Pi(G)$.

Theorem 12. A permutation $\pi \in B_{d}$ is permissible with respect to $G$ if and only if it satisfies the following condition:

If $(i, j)$ is an edge in $G$ and $+i$ appears in $\pi$, then $-j$ must precede $+i$ in $\pi$.

Proof. Suppose that $\mathbf{p}_{0}, \mathbf{p}_{1}, \mathbf{p}_{2}, \ldots, \mathbf{p}_{d}=\mathbf{z}$ is the sequence of points defining a maximal simplex $\sigma_{\pi} \subset \mathscr{P}(G)$. If $+i$ appears in $\pi$ and is not preceded by $-j$, then some of these points will have coordinates $x_{i}=2$ and $x_{j}=1$, so $x_{i}+x_{j}=3$, which is not allowed if $(i, j)$ is an edge in $G$. Conversely, a permutation satisfying the condition determines a maximal simplex $\sigma_{\pi}$ which is the convex hull of points whose coordinates satisfy the defining inequalities of $\mathscr{P}(G)$.

Suppose $K$ is a simplicial complex and $\mathscr{P}$ is a polytope (or any subset), both embedded in $\mathbf{R}^{n}$. By $K \cap \mathscr{P}$ we mean the subcomplex of $K$ contained in $\mathscr{P}$, assuming that this is well defined (which is always the case here).

For the remainder of this section, fix a graph $G$ and let $\mathscr{P}$ denote its extended 2-weak vertex-packing polytope. We define $\mathscr{P}$ to be the triangulation of $\mathscr{P}$ induced by the triangulation $\widehat{2 C^{d}}$ of $2 C^{d}$. That is, $\hat{\mathscr{P}}=\mathscr{P} \cap \widehat{2 C^{d}}$. We need to show, of course, that this actually triangulates $\mathscr{P}$, i.e., that $\hat{\mathscr{P}}$ is well defined.

Proposition 13. Let $\sigma_{\pi}$ be a maximal simplex in $c_{2}$. If $\mathscr{P}$ intersects the interior of $\sigma_{\pi}$, then $\sigma_{\pi} \subset \mathscr{P}$. Hence, $\hat{\mathscr{P}}=\widehat{2 C^{d}} \cap \mathscr{P}$ is a triangulation of $\mathscr{P}$.

Proof. Let $\mathbf{p}_{0}, \mathbf{p}_{1}, \mathbf{p}_{2}, \ldots, \mathbf{p}_{d}$ be the sequence of points defining $\sigma_{\pi}$ and suppose $(i, j)$ is an edge of $G$. Let $\pi=a_{1} a_{2} \cdots a_{d}$ and let $a_{k}$ be the first occurrence in $\pi$ of either $\pm i$ or $\pm j$. If $a_{k}>0$ (so $a_{k}$ equals $+i$ or $+j$ ), then every one of the points $\mathbf{p}_{m}$ satisfies $x_{i}+x_{j} \geq 2$ and if $a_{k}<0$, then all of the $\mathbf{p}_{m}$ satisfy $x_{i}+x_{j} \leq 2$. In either case the convex hull of the $\mathbf{p}_{m}$ 's, namely $\sigma_{\pi}$, lies entirely on one side of the hyperplane $H_{i, j}$ defined by $x_{i}+x_{j}=2$. Hence, $\sigma_{\pi}$ must lie entirely on one side of each of the hyperplanes defining $\mathscr{P}$, so either $\sigma_{\pi} \subset \mathscr{P}$ or else $\mathscr{P}$ intersects $\sigma_{\pi}$ only in the boundary of $\sigma_{\pi}$.

Remark 14. By Proposition 10, the triangulation $\hat{\mathscr{P}}$ of $\mathscr{P}$ is primitive. Hence, $\operatorname{Nvol}(\mathscr{P})=\# \Pi(G)$ and $h^{*}(\mathscr{P}, t)=h(\hat{\mathscr{P}}, t)$. 
Since $\mathscr{P}$ is triangulated by the simplical complex $\hat{\mathscr{P}}$, each vertex of $\mathscr{P}$ must be a vertex of a simplex in $\hat{\mathscr{P}}$, and thus have integral coordinates, since the vertices of the simplices $\sigma_{\pi}$ are clearly integral. However, we can do better than this. Namely, we can completely characterize all vertices of $\mathscr{P}$.

Theorem 15. Let $\mathbf{v}=\left(v_{1}, v_{2}, \ldots, v_{d}\right)$ be a point in $\mathscr{P}$ with integral coordinates (so $v_{i} \in\{0,1,2\}$ for all $i$ ) and let $S=\left\{i \in[d] \mid v_{i}=1\right\}$. Let $G_{S}$ be the subgraph of $G$ induced by $S$. Then $v$ is a vertes of $\mathscr{P}$ iff $S=\varnothing$ or each connected component of $G_{S}$ contains an odd cycle.

Proof. Suppose $S \neq \varnothing$ and that some component of $G_{S}$ contains no odd cycle. Call this component $C$ and let $T$ be its vertex set. Then $C$ is bipartite and thus two-colorable, so we can color the vertices of $C$ with the numbers 0 and 2 in such a way that no two adjacent vertices get the same color. Let $c(i)$ be the color of $i$. Define $\mathbf{x}=\left(x_{1}, x_{2}, \ldots, x_{d}\right)$ and $\mathbf{y}=\left(y_{1}, y_{2}, \ldots, y_{d}\right)$ by $x_{i}=y_{i}=v_{i}$ if $i \notin T$ and $x_{i}=c(i), y_{i}=2-c(i)$ if $i \in T$. Then $\mathbf{x}, \mathbf{y} \in \mathscr{P}$ and $\mathbf{v}=\frac{1}{2}(\mathbf{x}+\mathbf{y})$, so $\mathbf{v}$ is not a vertex of $\mathscr{P}$.

Conversely, suppose $S=\varnothing$ or that every component of $G_{S}$ contains an odd cycle. If $S=\varnothing$, then $\mathbf{v}$ is a vertex of $2 C^{d}$ and thus of $\mathscr{P}$. Assume then that $S \neq \varnothing$ and that every component of $G_{S}$ contains an odd cycle, so no component of $G_{S}$ is two-colorable. Assume also that $\mathrm{v}$ is not a vertex of $\mathscr{P}$. Then we must have $a$, $b$ with $0<a, b<1$, and $a+b=1$ and distinct points $\mathbf{x}, \mathbf{y} \in \mathscr{P}$ such that $a \mathbf{x}+$ $b \mathbf{y}=\mathbf{v}$. It is easy to see that $x_{i}=y_{i}=v_{i}$ for each $i$ such that $v_{i} \in\{0,2\}$. Hence, since $\mathbf{x}$ and $\mathbf{y}$ must be different from $\mathbf{v}$, there must be an $i$ such that $x_{i}, y_{i} \neq v_{i}=1$. Hence, $x_{i}>1$ or $y_{i}>1$, since otherwise $a x_{i}+b y_{i}<1=v_{i}$. Assume without loss of generality, that $x_{i}>1$. Now, $i$ belongs to some component of $G_{S}$ and that component contains an odd cycle, so there is a path beginning at $i$ and traveling through the entire cycle. Assume, without loss of generality, that the vertices in this path are labeled (in order) by $i, i+1, \ldots, i+m$. Since $x_{i}>1$, we have $x_{i+1}<1$, as the vertices $x_{i}$ and $x_{i+1}$ are adjacent, so $x_{i}+x_{i+1} \leq 2$. This means that $y_{i+1}>1$ since otherwise $a x_{i+1}+b y_{i+1}<1$. This implies that $y_{i+2}<1$, so $x_{i+2}>1$ and the argument can be repeated to show that $x_{i+r}>1$ iff $r$ is even. However, this amounts to a two-coloring of the path, including the odd cycle, which is a contradition, so $\mathbf{v}$ is indeed a vertex of $\mathscr{P}$.

Our goal is to find a shelling of $\hat{\mathscr{P}}$. To that end, we order the permutations in $B_{d}$ lexicographically, i.e., a permutation $\pi=a_{1} a_{2} \cdots a_{d}$ precedes $\tau=b_{1} b_{2} \cdots b_{d}$ if $a_{i}<b_{i}$ for the first $i$ at which $\pi$ and $\tau$ differ. Abusing notation, we use $<$ to denote this ordering of the elements of $B_{d}$. For example $\overline{2} 3 \overline{1}<3 \overline{2} \overline{1}$ and $23 \overline{1}<231$.

We will show that the ordering of maximal simplices in $\hat{P}$ induced by the lexicographic ordering of their corresponding permutations is a shelling of $\mathscr{P}$. We say that two $d$-simplices intersect maximally if they have a $(d-1)$-face in common.

Lemma 16. Suppose $\sigma_{\pi} \subset c_{z} \cap \widehat{\mathscr{P}}$, where $\pi=a_{1} a_{2} \cdots a_{d}$, and suppose that $i$ is $a$ descent in $\pi$. If $i$ is an internal descent in $\pi$, i.e., $a_{i}>a_{i+1}$, for some $i \leq d-1$, 
then $\sigma_{\pi^{\prime}} \subset c_{z} \cap \widehat{\mathscr{P}}$, where $\pi^{\prime}=a_{1} a_{2} \cdots a_{i+1} a_{i} \cdots a_{d}$. If $i=d$, i.e., $a_{d}>0$, then $\sigma_{\pi^{\prime}} \subset \hat{\mathscr{P}}$, where $\pi^{\prime}=a_{1} a_{2} \cdots-a_{d^{\prime}}$. In either case, $\pi^{\prime}<\pi$ and $\sigma_{\pi}$ and $\sigma_{\pi^{\prime}}$ intersect maximally. Moreover, if two maximal simplices $\sigma_{\pi}$ and $\sigma_{\pi}$ in $\widehat{\mathscr{P}}$ intersect maximally, then $\pi$ and $\pi^{\prime}$ either differ only by a single transposition or only by the sign of their last letter.

Proof. If $i<d$, so $\pi^{\prime}=a_{1} \cdots a_{i+1} a_{i} \cdots a_{d}$, then, by Lemma 9, $\sigma_{\pi} \subset c_{\mathrm{z}}$. If both $a_{i}$ and $a_{i+1}$ are negative, then there are no restrictions on the order in which they can appear in a permissible permutation. If both $a_{i}$ and $a_{i+1}$ are positive, then, since $\pi$ is permissible, $x_{a_{i}}+x_{a_{i+1}} \leq 2$ cannot be a defining inequality of $\mathscr{P}$, so again there are no restrictions on the order in which $a_{i}$ and $a_{i+1}$ can appear in a permissible permutation. Since $i$ is a descent, the only remaining possibility is $a_{i}>0$ and $a_{i+1}<0$. That implies that $\pi^{\prime}$ must be permissible if $\pi$ is.

If $i=d$, so $a_{d}>0$, then clearly $\pi^{\prime}=a_{1} a_{2} \cdots-a_{d}$ is permissible if $\pi$ is.

Now, regardless of whether $i<d$ or $i=d$, suppose that $\mathbf{p}_{0}, \mathbf{p}_{1}, \ldots, \mathbf{p}_{d}$ is the sequence of points defining $\sigma_{\pi}$. Then the sequence defining $\sigma_{\pi^{\prime}}$ is $\mathbf{q}_{0}, \mathbf{q}_{1}, \ldots, \mathbf{q}_{d}$, where $\mathbf{q}_{j}=\mathbf{p}_{j}$, except for $j=i$. Hence, $\sigma_{\pi}$ and $\sigma_{\pi^{\prime}}$ intersect in the $(d-1)$-face defined by the convex hull of the points $\mathbf{p}_{0}, \mathbf{p}_{1}, \ldots, \mathbf{p}_{i-1}, \mathbf{p}_{i+1}, \ldots \mathbf{p}_{d}$ (where possibly $i=d$ ).

If two maximal simplices intersect maximally, then their defining sequences of points differ by just one point. It is then straightforward to check that their corresponding permutations can differ only as described.

Theorem 17. Order the maximal simplices in $\hat{\mathscr{P}}$ so that $\sigma_{\tau}$ precedes $\sigma_{\pi}$ if $\tau<\pi$. This ordering is a shelling of $\hat{\mathscr{P}}$.

Proof. Let $\sigma_{\pi}$ be a maximal simplex in $\hat{\mathscr{P}}$. If $\pi$ is the (lexicographically) first permutation in $\Pi(G)$, then there is nothing to prove. Otherwise, we must show that $\sigma_{\pi} \cap \bigcup_{\tau<\pi} \sigma_{\tau}$ is a nonempty union of $(d-1)$-faces of $\sigma_{\pi}$. It suffices to show that if $\sigma_{\pi}$ intersects a maximal simplex $\sigma_{\tau} \subset \widehat{\mathscr{P}}$ and $\sigma_{\tau}$ precedes $\sigma_{\pi}$, then $\sigma_{\pi} \cap \sigma_{\tau}$ is contained in some $(d-1)$-face $f$ of $\sigma_{\pi}$ such that $f=\sigma_{\pi} \cap \sigma_{\pi^{\prime}}$ for some $\sigma_{\pi^{\prime}} \subset \hat{\mathscr{P}}$ with $\sigma_{\pi^{\prime}}$ preceding $\sigma_{\pi}$.

Suppose $\sigma_{\pi}, \sigma_{\tau} \subset \hat{\mathscr{P}}$ and that $\sigma_{\tau}$ precedes $\sigma_{\pi}$, so $\tau<\pi$. Let $i$ be the first place where $\pi$ and $\tau$ differ. If $i=d$, then $\sigma_{\pi}$ and $\sigma_{\tau}$ intersect maximally, by Lemma 16, and we are done. Assume therefore that $i<d$. Let $\pi=a_{1} a_{2} \cdots a_{d}$ and $\tau=$ $a_{1} a_{2} \cdots a_{i-1} b_{i} \cdots b_{d}$. Let $k$ be the first descent in $\pi$ after $i-1$. Such a $k$ must exist, because otherwise we would have $a_{i}<a_{i+1}<\cdots<a_{d}<0$ so that $\pi$ was the first permuation in $B_{d}$ beginning with $a_{1} a_{2} \cdots a_{i-1}$, contradicting $\tau<\pi$.

Let $\mathbf{p}_{0}, \mathbf{p}_{1}, \ldots, \mathbf{p}_{\mathbf{d}}$ be the sequence of points defining $\sigma_{n}$. We claim that $\mathbf{p}_{k} \notin \sigma_{\mathfrak{r}}$. If $\sigma_{\tau}$ did contain $\mathbf{p}_{k}$, then we would have $\left\{a_{1}, a_{2}, \ldots, a_{k}\right\}=\left\{b_{1}, b_{2}, \ldots, b_{k}\right\}$, in particular $\left\{a_{i}, a_{i+1}, \ldots, a_{k}\right\}=\left\{b_{i}, b_{i+1}, \ldots, b_{k}\right\}$, so $k>i$. However, then, since $k$ was the first descent in $\pi$ after $i-1$, so that $a_{i}<a_{i+1}<\cdots<a_{k}$, we must have $b_{i}>a_{i}$, contradicting the assumption $\tau<\pi$, so $\mathbf{p}_{k} \notin \sigma_{\tau}$.

If $k<d$, let $\pi^{\prime}=a_{1} \cdots a_{k+1} a_{k} \cdots a_{d}$. Then $\pi^{\prime}<\pi$ and $\sigma_{\pi} \cap \sigma_{\pi^{\prime}}$ is the convex hull of $\mathbf{p}_{0}, \mathbf{p}_{1}, \ldots, \mathbf{p}_{k-1}, \mathbf{p}_{k+1}, \ldots, \mathbf{p}_{d}$. By Lemma $16, \sigma_{\pi}$ and $\sigma_{\pi^{\prime}}$ intersect maximally and $\sigma_{\pi^{\prime}} \subset \mathscr{P}$. Moreover, since $\mathbf{p}_{k} \notin \sigma_{\tau}, \sigma_{\pi} \cap \sigma_{\tau} \subset \sigma_{\pi} \cap \sigma_{\pi^{\prime}}$, as desired.

If $k=d$, then $a_{d}>0$. Let $\pi^{\prime}=a_{1} a_{2} \cdots-a_{d}$. Then $\pi^{\prime}<\pi$ and, by Lemma $16, \sigma_{\pi}$ 
and $\sigma_{\pi^{\prime}}$ intersect maximally and $\sigma_{\pi^{\prime}} \subset \widehat{\mathscr{P}}$. Since $\mathbf{p}_{d} \notin \sigma_{\tau}$, we have $\sigma_{\pi} \cap \sigma_{\tau} \subset \sigma_{\pi} \cap \sigma_{\pi^{\prime}}$, as desired.

Theorem 18. The h-polynomial of $\hat{\mathscr{P}}$ equals the descent polynomial of the set of permissible permutations with respect to $G$. That is, $h(\hat{\mathscr{P}}, t)=D(\Pi(G), t)$.

Proof. By Theorem 3, we need to show that for each descent in $\pi \in \Pi(G)$ there is a unique maximal simplex $\sigma_{\tau} \in \hat{\mathscr{P}}$ such that $\sigma_{\pi}$ and $\sigma_{\tau}$ intersect maximally and such that $\tau<\pi$. First suppose that $i$ is an internal descent in $\pi$, i.e., $1 \leq i \leq d-1$ and let $\pi=a_{1} a_{2} \cdots a_{d}$, so $a_{i}>a_{i+1}$. By Lemma 16, two maximal simplices $\sigma_{\pi}$ and $\sigma_{\mathrm{r}}$ in the same cube $c_{\mathbf{z}}$ intersect maximally if and only if $\pi$ and $\tau$ differ by a single transposition. Let $\tau=a_{1} a_{2} \cdots a_{i+1} a_{i} \cdots a_{d}$. Then $\tau$ precedes $\pi, \sigma_{\tau} \subset \hat{\mathscr{P}}$, and $\sigma_{\pi}$ and $\sigma_{\mathrm{r}}$ intersect maximally. Conversely, if $\sigma_{\pi}$ and $\sigma_{\tau}$ in $c_{\mathrm{z}}$ intersect maximally, then they differ by a single transposition and if $\tau<\pi$, then $\pi$ has a descent between the two letters involved in that transposition.

The only other maximal simplices $\sigma_{\pi}$ can intersect maximally are those belonging to cubes other than $c_{\mathbf{z}}$. By Lemma 16 , if $\sigma_{\tau}$ is such a simplex and $\pi=a_{1} a_{2} \cdots a_{d}$, then $\tau=a_{1} a_{2} \cdots-a_{d}$, so, for $\tau$ to precede $\pi$, we must have $a_{d}>0$, i.e., $d$ is a descent in $\pi$. Conversely, if $d$ is a descent in $\pi$, then $a_{d}>0$, so if $\tau=a_{1} a_{2} \cdots-a_{d}$, then $\tau<\pi, \sigma_{\tau} \subset \hat{\mathscr{P}}$, and $\sigma_{\pi}$ and $\sigma_{\tau}$ intersect maximally.

\section{Applications}

As we are much concerned with that part of the boundary of $\mathscr{P}$ which is contained in $\partial\left(2 C^{d}\right)$, we make the following definition. (Unless otherwise specified, $\mathscr{P}$ and $\hat{\mathscr{P}}$ have the same meaning as before.)

Definition 19. $\mathscr{B}:=\mathscr{P} \cap \partial\left(2 C^{d}\right)$ and $\hat{\mathscr{B}}:=\hat{\mathscr{P}} \cap \partial\left(2 C^{d}\right)$. A facet of $\mathscr{B}$ is a facet of $\mathscr{P}$ contained in $\mathscr{B}$.

Theorem 20. $h(\hat{\mathscr{P}}, t)=h(\widehat{\mathscr{B}}, t)$. Hence, $h^{*}(\mathscr{P}, t)=h(\hat{\mathscr{B}}, t)=h^{*}(\mathscr{B}, t)$.

Proof. $\hat{\mathscr{P}}$ is a cone over $\hat{\mathscr{B}}$, which yields the equality of $h$-polynomials, by Theorem 4. The latter two equalities are then implied by Theorem 1 and the fact that $\widehat{\mathscr{P}}$ (and hence $\hat{\mathscr{B}}$ ) is primitively triangulated.

Corollary 21. $\operatorname{vol}_{d}(\mathscr{P})=\operatorname{vol}_{d-1}(\mathscr{B})$. Equivalently, $\operatorname{Nvol}(\mathscr{P})=\operatorname{Nvol}(\mathscr{B})$

Proof. Nvol $(\mathscr{P})$ equals the number of maximal simplices in $\widehat{\mathscr{P}}$, which in turn equals the number of maximal simplices in $\hat{\mathscr{B}}$, since $\hat{\mathscr{P}}$ is a cone over $\hat{\mathscr{B}}$.

Corollary 21 yields a recursive formula for the volume of $\mathscr{P}$, because each facet of $\mathscr{B}$ is an extended 2-weak vertex-packing polytope. More precisely, the facet of obtained by setting $x_{i}=0$ (which we denote $\mathscr{B}_{x_{i}=0}$ ) is the extended 2-weak 
vertex-packing polytope of the graph obtained by removing $x_{i}$ from $G$. If $x_{i}$ is an isolated vertex of $G$, then $\mathscr{B}_{x_{i}=2}$ is isometric to $\mathscr{B}_{x_{i}=0}$ (since then $\mathscr{P}=$ $\mathscr{P}_{x_{i}=0} \times[0,2]$ ), but otherwise $\mathscr{B}_{x_{i}=2}$ has dimension less than $d-1$ and thus $\operatorname{vol}_{d-1}\left(\mathscr{R}_{x_{1}=2}\right)=0$.

If $d=a_{1}+a_{2}+\cdots+a_{k}$, let $\left(\begin{array}{c}d \\ a_{1}, a_{2}, \ldots, a_{k}\end{array}\right)$ be the multinomial coefficient defined by

$$
\left(\begin{array}{c}
d \\
a_{1}, a_{2}, \ldots, a_{k}
\end{array}\right)=\frac{d !}{a_{1} ! a_{2} ! \cdots a_{k} !}
$$

Abusing notation, in what follows, we write $\operatorname{Nvol}(G)$ instead of $\operatorname{Nvol}(\mathscr{P}(G)$ ), where $G$ is a graph and $\mathscr{P}(G)$ is its extended 2-weak vertex-packing polytope.

Theorem 22. Let $C_{1}, C_{2}, \ldots, C_{k}$ be the connected components of $G$, with $a_{i}=\# C_{i}$ for each $i$, and $d=\# G$. Then

$$
\operatorname{Nvol}(G)=\left(\begin{array}{c}
d \\
a_{1}, a_{2}, \ldots, a_{k}
\end{array}\right) \prod_{i=1}^{k} \operatorname{Nvol}\left(C_{i}\right)
$$

In particular, if $G$ has an isolated vertex $x$ and $G_{x}$ is the graph obtained by removing $x$ from $G$, then $\operatorname{Nvol}(G)=2 \cdot d \cdot \operatorname{Nvol}\left(G_{x}\right)$.

Proof. For the sake of simplicity, assume that $G$ has two connected components, $C_{1}$ and $C_{2}$, with vertices $x_{1}, x_{2}, \ldots, x_{m}$ and $x_{m+1}, x_{m+2}, \ldots, x_{d}$, respectively. A permutation $\pi$ is permissible with respect to $G$ if and only if the subword of $\pi$ corresponding to each component is permissible with respect to that component. Hence, given permutations $\pi_{1} \in \Pi\left(C_{1}\right)$ and $\pi_{2} \in \Pi\left(C_{2}\right),{ }^{1}$ every "shuffling" of $\pi_{1}$ and $\pi_{2}$ is a permissible permutation with respect to $G$. That is, we can select any $m$ positions out of $1,2, \ldots, d$ and place the letters of $\pi_{1}$, preserving their order, in these positions and the letters of $\pi_{2}$, preserving their order, in the remaining $d-m$ positions. This means that

$$
\# \Pi(G)=\left(\begin{array}{l}
d \\
m
\end{array}\right) \cdot \# \Pi\left(C_{1}\right) \cdot \# \Pi\left(C_{2}\right)
$$

and the argument obviously generalizes to any number of components with $\left(\begin{array}{l}d \\ m\end{array}\right)$ replaced by the multinomial coefficient $\left(\begin{array}{c}d \\ a_{1}, a_{2}, \ldots, a_{k}\end{array}\right)$.

\footnotetext{
${ }^{1}$ We are being a little sloppy with the notation here.
} 
In view of the preceding theorem, we can now restrict our attention to connected graphs, in particular to graphs without isolated vertices.

Theorem 23. Let $G$ be a graph without isolate vertices, \# $G=d$, and let $G_{x}$ denote the graph obtained by removing the vertex $x$ from $G$. Then

$$
\operatorname{Nvol}(G)=\sum_{x \in G} \operatorname{Nvol}\left(G_{x}\right)
$$

Proof. As pointed out above, if $G$ has no isolated vertices, then all the facets of $\mathscr{B}=\mathscr{P} \cap 2 C^{d}$ are of the form $\mathscr{B}_{x_{t}=0}$ and so $\mathscr{B}=\bigcup_{x \in G} \mathscr{B}_{x=0}$. Clearly, $\operatorname{Nvol}(\mathscr{B})=$ $\sum_{x \in G} \operatorname{Nvol}\left(\mathscr{B}_{x=0}\right)$, which, together with Corollary 21 , yields the theorem.

We now give a few examples of how the recurrence affored by Theorems 22 and 23 can be used to compute the volume of extended 2-weak vertex-packing polytopes. To get the recursion off the ground, observe that if $G$ consists of a single vertex, then $\mathscr{P}(G)=[0,2] \subset \mathbf{R}$, so $\operatorname{Nvol}(G)=2$.

\section{Example 24.}

$$
\begin{aligned}
\operatorname{Nvol}(\bullet \bullet \bullet) & =2 \cdot \operatorname{Nvol}(\bullet \bullet)+\operatorname{Nvol}(\bullet \bullet) \\
& =2 \cdot 2 \cdot \operatorname{Nvol}(\bullet)+\left(\begin{array}{l}
2 \\
1
\end{array}\right) \cdot(\operatorname{Nvol}(\bullet))^{2} \\
& =8+2 \cdot 2^{2}=16 .
\end{aligned}
$$

Example 25.

$$
\begin{aligned}
\operatorname{Nvol}(\bullet \bullet) & =2 \cdot \operatorname{Nvol}(\bullet \bullet \bullet)+2 \cdot \operatorname{Nvol}(\bullet) \\
& =2 \cdot 16+6 \cdot \operatorname{Nvol}(\bullet \bullet)=32+6 \cdot 4=56
\end{aligned}
$$

Example 26. If $G_{d}$ is the graph $\multimap-\cdots \rightarrow-$ with $d$ vertices, so $G_{d}$ is the comparability graph of the fence poset on elements $x_{1}, \ldots, x_{d}$ (with relations $x_{1}<x_{2}>x_{3}<x_{4}>\cdots$ ), then it is well known (see [12]) that the volume of the 2-weak vertex-packing polytope $\mathscr{W}\left(G_{d}\right)$ of $G_{d}$ is given by the $d$ th coefficient of the Taylor series of $\tan x+\sec x$. We can compute the corresponding result for $\mathscr{P}\left(G_{d}\right)$ in the following way. Using Theorems 22 and 23, we get this recurrence for $A_{d}=\operatorname{Nvol}\left(G_{d}\right):$ For $d \geq 1$,

$$
A_{d+1}=\sum_{i=0}^{d}\left(\begin{array}{l}
d \\
i
\end{array}\right) A_{i} \cdot A_{d-i} \text { and hence } \sum_{d \geq 1} A_{d+1} \frac{x^{d}}{d !}=\left(\sum_{d \geq 0} A_{d} \frac{x^{d}}{d !}\right)^{2}
$$


Setting $F(x)=\sum_{d \geq 0} A_{d}\left(x^{d} / d !\right)$ yields $F^{\prime}(x)-1=(F(x))^{2}$, which, together with $F(0)=1$, has the unique solution $F(x)=\tan (2 x)+\sec (2 x)$.

We give one example of how $h(\hat{\mathscr{P}}(G), t)$ can be computed from $\Pi(G)$.

Example 27. Let $K_{d}$ be the complete graph on $d$ vertices. Then $\Pi\left(K_{d}\right)$ consists of all permutations $\pi=a_{1} a_{2} \cdots a_{d} \in B_{d}$ such that $a_{i}<0$ for all $i$ except, perhaps, for $i=d$. Let $\Pi_{-}$be the set of permutations in $\Pi(G)$ whose letters are all negative, and let $\Pi_{+}$be the set of those permutations in $\Pi(G)$ whose last letter is positive (but all others negative). It is easy to see that $D\left(\Pi_{-}, t\right)$ equals the usual descent polynomial of the symmetric group $S_{d}$, consisting of all permutations of the letters $\{1,2, \ldots, d\}$ (no signs involved and never a descent at $d$ ). This polynomial is well known and is called the $d$ th Eulerian polynomial (see, e.g., [11]) and often denoted by $A_{d}(t){ }^{2}$ In fact, $A_{d}(t)$ equals the $h$-polynomial of our triangulation of $c_{0}$. As for $\Pi_{+}$, we see that there is always a descent at $d$, never a descent at $d-1$, and the first $d-1$ letters in a $\pi \in \Pi_{+}$behave just like a permutation in $S_{d-1}$. Hence each permutation in $\Pi_{+}$corresponds to a permutation in $S_{d-1}$, but has an extra descent, namely the one at $d$. There are $d$ possible choices for the last letter of $\pi \in \Pi_{+}$and the descent polynomial of $\Pi_{+}$is thus equal to $d \cdot t A_{d-1}(t)$. Hence, $h\left(\hat{\mathscr{P}}\left(K_{d}\right), t\right)=$ $A_{d}(t)+d \cdot t A_{d-1}(t)$.

Note also that $\operatorname{Nvol}\left(K_{d}\right)=\# \Pi\left(K_{d}\right)=2 \cdot d !$ This volume was previously computed by Lee and Morris [5].

Finally, we record, without proof, two theorems which give a recursive algorithm for computing $h(\hat{\mathscr{P}}, t)$. For proofs, see Theorems 38 and 35 in [15], which also contains many examples and details not found here. Recall that $[d]=$ $\{1,2, \ldots, d\}$.

Definition 28. Let $G$ be a graph with vertex set $[d]$ and let $\mathscr{P}$ be defined as usual in terms of $G$. Let $S \subset[d]$. Then $\mathscr{P}_{S}:=\left\{\mathbf{x}=\left(x_{1}, x_{2}, \ldots, x_{d}\right) \in \mathscr{P} \mid x_{i}=0\right.$ if $\left.i \in S\right\}$.

That is, $\mathscr{P}_{S}$ is isomorphic to $\mathscr{P}\left(G_{S}\right)$, where $G_{S}$ is the subgraph of $G$ induced by $[d] \backslash S$. We also define $\widehat{\mathscr{P}_{S}}$ similarly, i.e., $\widehat{\mathscr{P}_{S}}:=\widehat{\mathscr{P}} \cap \mathscr{P}_{S}$.

Theorem 29. Let $G$ be a graph with vertex set [d] and no isolated vertices. Then $h(\hat{\mathscr{P}}, t)=\sum s h\left(\hat{\mathscr{P}}_{S}, t\right)(t-1)^{* S-1}$, where $S$ ranges over all nonempty subsets of $[d]$.

Theorem 30. Let $G$ be a graph with $d-1$ vertices and denote by $G^{\prime}$ the graph obtained by adding an isolated vertex to $G$. Suppose $h(\hat{P}(G), t)=a_{0}+a_{1} t+\cdots$ $+a_{d-1} t^{d}$. Then $h\left(\hat{\mathscr{P}}\left(G^{\prime}\right), t\right)=b_{0}+b_{1} t+\cdots+b_{d} t^{d}$, where

$$
b_{k}=(2 k+1) a_{k}+(2 d-2 k+1) a_{k-1} .
$$

\footnotetext{
${ }^{2}$ Usually, though, $A_{d}(t)$ is defined so that $D\left(\Pi_{-}, t\right)=t^{-1} A_{d}(t)$.
} 


\section{References}

1. U. Betke and P. McMullen: Lattice points in lattice polytopes, Monatsh. Math. 99 (1985), 253-265.

2. A. Björner: Shellable and Cohen-Macaulay partially ordered sets, Trans. Amer. Math. Soc. 260 (1980), 159-183.

3. A. Björner: Topological methods, Handbook of combinatorics (R. Graham, M. Grötschel, and L. Lovász, eds.), North-Holland, Amsterdam, to appear.

4. V. Chvátal: On certain polytopes associated with graphs, J. Combin. Theory Ser. B 18 (1975), $138-154$.

5. J. Lee and W. D. Morris, Geometric comparison of combinatorial polytopes, CORE Discussion Paper 9216, 1992.

6. P. McMullen: The maximum numbers of faces of a convex polytope. Mathematika 17 (1970), 179-184.

7. J. Munkres: Elements of Algebraic Topology, Addison-Wesley, Menlo Park, CA, 1984.

8. M. Padberg: On the facial structure of set packing polyhedra, Math. Programming 5 (1973), $199-215$.

9. V. Reiner: Signed permutation statistics, European J. Combin. 14 (1993), 553-567.

10. R. Stanley: Decomposition of rational convex polytopes, Ann. Discrete Math. 6 (1980), 333-342.

11. R. Stanley: Enumerative Combinatorics, vol. I. Wadsworth \& Brooks/Cole, Pacific Grove, CA, 1986.

12. R. Stanley: Two poset polytopes, Discrete Comput. Geom. 1 (1986), 9-23.

13. R. Stanley: Generalized $h$-vectors, intersection cohomology of toric varieties, and related results, Commutative Algebra and Combinatorics, Advanced Studies in Pure Mathematics, Vol 11, 1987, pp. 187-213.

14. E. Steingrimsson: Permutation statistics of indexed and poset permutations, Ph.D. thesis, Massachusetts Institute of Technology, 1991.

15. E. Steingrímsson: Weak vertex-packing polytopes, Preprint 1993:19, Matematiska Institutionen CTH \& GU.

Received May 13, 1993, and in revised form December 16, 1993, and February 7, 1994. 